\title{
EDITORIAL
}

\section{The quest for better diagnosis: DSM-5 or RDoC?}

\author{
Antonio Egidio Nardi, ${ }^{1}$ Flavio Kapczinski, ${ }^{2}$ João Quevedo, ${ }^{1}$ Jaime E.C. Hallak, ${ }^{1}$ Rafael Freire, ${ }^{3}$ \\ Marco Aurélio Romano-Silva ${ }^{1}$ \\ ${ }^{1}$ Associate Editors, ${ }^{2}$ Editor-in-Chief, and ${ }^{3}$ Junior Editor of Revista Brasileira de Psiquiatria.
}

Diagnosis in psychiatry has never been an easy task. Every psychiatrist or researcher struggles with diagnostic limitations on a daily basis. The mixture of symptoms, behavior, culture, prejudice, and science has always been the subject of the most enthusiastic debate, but with very few results. The release of the Research Diagnostic Criteria (RDC) ${ }^{1}$ in 1972, soon followed by the DSM-III, and their diagnostic criteria, lent greater reliability to psychiatric diagnosis, but yielded almost no gains in validity.

We are now in the dawn of the new edition of the Diagnostic and Statistical Manual of Mental Disorders (DSM-5). It is the result of long debate, consensus, and research data collected over the last two decades. Even though we may criticize some of its aspects and support others, the great development observed in clinical research after the third and fourth editions of the DSM cannot be denied. The main principle of DSMs is that they are important tools for research and legal work. For clinicians, they serve as guidelines only, and should be no more than that. Clinical practice is an art in which we have to mix science and different levels of philosophy.

Thomas Insel, ${ }^{2}$ director of the National Institute for Mental Health (NIMH), has recently published an editorial stating that the greatest provider of funds for mental health research will not accept the DSM-5 as a valid tool for research. This statement creates an uncomfortable situation for the American Psychiatric Association at this moment, when the DSM- 5 is being presented to the world as the basis for the most modern and reliable diagnoses.

DSM-5 categories are not supposed to be a perpetual "gold standard." First, they represent a set of contemporary criteria for an accurate diagnosis that can and will change in the next edition of the manual. Second, the use of the DSM-5 does not weaken the Research Domain Criteria (RDoC) project. In fact, the two publications could work together and mutually support each other. Any diagnostic system should be based on emerging research data, but symptom-based categories are what we currently have. We certainly agree with Insel in that research needs to gather genetic, imaging, physiologic, and cognitive evidence in order to improve our understanding of how all biological data - rather than symptoms alone - cluster and how these clusters relate to treatment response. We can already identify some of these issues in the DSM-5. Some new categories may not be sufficiently sound from the scientific point of view. For example, mild neurocognitive disorder, binge eating disorder, and disruptive mood dysregulation disorder are all very close to normal behavior, and we still lack clear cut-off points to improve diagnostic reliability. Some other categories had their formerly strict criteria changed to more open possibilities. No diagnostic classification will address all human needs in this regard, and it must be borne in mind that not all problems in life are caused by mental disorders.

NIMH will re-orient its research away from DSM categories. ${ }^{2}$ According to Insel, patients with mental disorders deserve better. But what could be better than DSM-5 at present? Insel's editorial informs that the NIMH has released the RDoC "to transform diagnosis by incorporating genetics, imaging, cognitive science, and other levels of information to lay the foundation for a new classification system." The NIMH tried to create a new nosology based on five major systems: 1) negative valence systems; 2) positive valence systems; 3) cognitive systems; 4) systems for social processes; and 5) arousal/modulatory systems. Even though Insel declares that at present we cannot design a system that is based on biomarkers or cognitive performance because we lack the data, symptoms and long-term follow-up is what physicians have today at their desk to support diagnosis. The data required for a precise diagnosis - one that we can really trust - will come in the future, but the patient is suffering now.

$\mathrm{NIMH}^{2}$ will support research projects that look across current categories - or that subdivide current categories to begin to develop a better system. Accordingly, clinical trials should enroll all patients receiving treatment in a given mood disorders clinic, rather than only those meeting strict major depressive disorder criteria. Likewise, studies of biomarkers for depression could begin by looking across many disorders associated with anhedonia or emotional appraisal bias or psychomotor retardation to understand the circuitry underlying these symptoms. $^{2}$

The RDoC project, for now, is a research framework, not a clinical tool. It is a decade-long project that is just beginning. We hope that RDoC will bring much valuable data, but right now it is a plan to transform clinical practice by bringing forth a new body of research to inform how we should diagnose and treat mental disorders. ${ }^{2}$

Working with psychiatric diagnosis means dealing with and searching for all the possibilities to improve future classifications. The RDoC and the DSM are not opposite to each other; they are different pathways by which 
psychiatry can move to improve diagnosis. ${ }^{3}$ Research should be our priority in obtaining data, as research data will be our basis to help people with mental disorders. Today, physicians should be aware of the limitations of our classifications, but also that they are the best we have at the moment. As for the future, we need robust research data and less fear of challenging psychiatric diagnosis.

\section{Disclosure}

Antonio Egidio Nardi has received grants from Conselho Nacional de Desenvolvimento Científico e Tecnologico (CNPq), Fundação Fundação de Amparo à Pesquisa do Estado do Rio de Janeiro (FAPERJ), and National Science and Technology Institute for Translational Medicine (INCT-TM); and has participated in the scientific board and as a speaker for Lundbeck, F. Hoffmann La-Roche, and GSK. Flavio Kapczinski has received grants/ research support from Astra-Zeneca, Eli Lilly, JanssenCilag, Servier, CNPq, Coordenação de Aperfeiçoamento de Pessoal de Nível Superior (CAPES), NARSAD, and Stanley Medical Research Institute; has been a member of the board of speakers for Astra-Zeneca, Eli Lilly, Janssen, and
Servier; and has served as a consultant for Servier. João Quevedo has received grants from CNPq and Fundação de Amparo à Pesquisa do Estado de Santa Catarina (FAPESC). Jaime E.C. Hallak has received grants from CNPq, Fundação Fundação de Amparo à Pesquisa do Estado de São Paulo (FAPESP), and INCT-TM. Rafael Freire has received post-doctoral grants from CNPq. Marco Aurelio Romano-Silva has received grants/research support from CNPq, CAPES, and Fundação de Amparo à Pesquisa do Estado de Minas Gerais (FAPEMIG).

\section{References}

1 Feighner JP, Robins E, Guze SB, Woodruff RA, Winokur G, Munoz R. Diagnostic criteria for use in psychiatric research. Arch Gen Psychiatry. 1972;26:57-63.

2 Insel T. National Institute of Mental Health: Director's Blog [Internet]. Transforming diagnosis. 2013 Apr 29 [cited 2013 May 16]. http://www. nimh.nih.gov/about/director/2013/transforming-diagnosis.shtml/

3 Jabr F. Scientific American Blogs: Brainwaves [Internet]. No one is abandoning the DSM, but it is almost time to transform it. 2013 May 7 [cited 2013 May 16]. http://blogs.scientificamerican.com/ brainwaves/2013/05/07/no-one-is-rejecting-the-dsm-but-it-is-almosttime-to-transform-it/ 